\title{
Trayectorias laborales y educativas de jóvenes racializados en Catalunya desde una perspectiva interseccional
}

\section{Labor and educational trajectories of racialized youth in Catalonia. Reflections from an intersectional perspective.}

\section{Josselyn Urdiales Correa ${ }^{1}$}

Universidad de Barcelona, España.

\section{Rosa Lázaro Castellanos}

Universidad de Barcelona, España.

Recibido: 29/03/2021

Aceptado: 04/06/2021

\section{Cómo citar}

Urdiales, J. y Lázaro, R. (2021). Trayectorias laborales y educativas de jóvenes racializados en Catalunya desde una perspectiva interseccional. Propuestas Críticas en Trabajo Social Critical Proposals in Social Work 1(2), 97-119. DOI: 10.5354/2735-6620.2021.60948

\section{Resumen}

La inmigración española de la década de los noventa trajo consigo transformaciones sociales, educativas y laborales para la población en general y, en particular, retos para las y los hijos de familias inmigrantes. Después de tres décadas, se suele sostener que la integración social, educativa y laboral de los hijos de inmigrantes es favorable, aunque distinta a la de los jóvenes autóctonos. El presente

Palabras Clave: Interseccionalidad; jóvenes; racialización; trayectorias educativas; trayectorias laborales 
artículo abordará estas distinciones, asumiendo una perspectiva interseccional para analizar las trayectorias educativas y laborales de jóvenes de origen inmigrante y racializados en Barcelona. A partir de una investigación cualitativa centrada en las trayectorias laborales de estos jóvenes mostraremos las restricciones que la clase, el género y el origen tienen en su promoción profesional dentro de un mercado laboral cada vez más competitivo y precario.

\section{Abstract}

Spanish immigration in the 1990s brought with it social, educational and labor transformations for the population in general and, in particular, challenges for

Keywords: Intersectionality; young people; racialization; educational trajectories; labor trajectories

\section{Introducción}

El Estado español cuenta con una población de origen extranjero de 4,5 millones, siendo Catalunya la Comunidad Autonómica que más población recibe. Del total de la población, 30\% presenta origen latinoamericano (Mahía, 2018). Es un perfil joven, en edad laboral y liderada por mujeres (Pedone, 2010). Además, en España uno de cada cuatro jóvenes menores de 18 años es descendiente de padres extracomunitarios (Universidad Carlos III de Madrid, 2019).

Las y los jóvenes de origen inmigrante y racializados son percibidos como una posible contribución al avance económico y cultural de los países de destino, pero también como generadores de desafíos. Para las familias de origen inmigrante la escolarización también tiene un peso importante, se proyecta sobre ella el ascenso social de los hijos/ as nacidos o crecidos en los países de acogida (Portes et al., 2018, p. 150). Socialmente, 
se apela a la inserción educativa y laboral de las y los jóvenes como posibles vías hacia una integración positiva.

Pese a los esfuerzos, la promoción educativa y laboral de los hijos de inmigrantes es una tarea pendiente en la mayoría de los países receptores de inmigración. Según la Investigación Longitudinal sobre la "Segunda Generación" en España (Portes et al., 2018), el número de hijos de inmigrantes que llegan a la Universidad no pasa el 30\%; además una proporción significativa de las y los jóvenes ocupa empleos precarizados. Por tanto, las trayectorias educativas y laborales exitosas dependen de un conjunto de estructuras sociales, ambientales o contextuales, las cuales pueden llegar a condicionar las trayectorias.

Partimos del supuesto que las y los jóvenes inmigrados y racializados, aunque son nacionales, comparten con sus progenitores experiencias de racismo, discriminación y desigualdades que son estructurales e históricas. El presente artículo tiene como objetivo conocer las percepciones sobre el sexismo, clasismo y racismo identificados por las y los jóvenes inmigrados y racializados con estudios superiores finalizados. Además, identificaremos los factores que limitan o facilitan sus trayectorias educativas y laborales.

Emplearemos la interseccionalidad como perspectiva de análisis para hacer visibles las desigualdades y dificultades que enfrentan las y los jóvenes migrados y racializados con estudios superiores finalizados, porque es una perspectiva que fomenta una comprensión de la desigualdad basada en las interacciones entre diversos sistemas de opresión, reconociendo que los factores que causan las exclusiones sociales raramente dependen de un único factor (Hill y Bilge, 2019).

El artículo está dividido en tres apartados, el primero se centra en la interseccionalidad como una herramienta útil para hacer visible la articulación de múltiples opresiones y propicia nuevos marcos reflexivos que contribuyan a desestabilizar las estructuras de poder. El segundo apartado hace referencia a la metodología empleada en la investigación. Un tercer apartado presenta los resultados de la investigación, aquí veremos cómo la articulación de la clase, el género y la racialización perjudican las trayectorias laborales y educativas de los jóvenes migrados y racializados. Y finalizamos con unas breves conclusiones. 


\section{La interseccionalidad}

La interseccionalidad es un concepto desarrollado por Kimberly Crenshaw (1989), para mostrar que la articulación clase, género y origen tiene repercusiones materiales y en la subjetividad de las mujeres negras y latinas. La autora estudia el caso de la General Motors, compañía que fue demandada por un grupo de mujeres negras, quienes sostenían ser discriminadas por la empresa. Sin embargo, el caso fue desestimado legalmente, pues la empresa presumía que no había discriminación por género, ni por motivos raciales, porque tenía contratadas mujeres blancas y a hombres negros.

Para Crenshaw (1989), existían dos discriminaciones interrelacionadas que no experimentaban los hombres negros, ni las mujeres blancas, es decir, nos muestra desigualdades y violencias concretas experimentadas por las mujeres negras, omitidas por la norma jurídica. Por tanto, la interseccionalidad permite pensar a las mujeres como un grupo heterogéneo, reconocer las diferencias entre las mujeres y el impacto de los distintos sistemas de opresión, difíciles de observar para las feministas blancas de clase media (Davis, 2005), cuyo privilegio de clase y raza las ha llevado a centrarse en la opresión de género.

La imbricación de las violencias experimentadas por distintas personas y colectivos fue denunciada desde los años setenta por las feministas negras (Brah, 2011; Davis, 2005). Señalaban que la lucha feminista no podía centrarse en un solo eje de desigualdad, porque dejaba fuera a las mujeres excluidas por los distintos sistemas de opresión (Hill y Bilge, 2019), por ejemplo, a las mujeres negras, de clases empobrecidas, indígenas, gitanas e inmigrantes. Mujeres colocadas en un estatus de inferioridad social, política y económica.

Por tanto, las violencias y discriminaciones imbricadas experimentadas por las mujeres, han sido nombradas en distintos contextos, sin llamarle interseccionalidad. Por ejemplo, las múltiples opresiones aparecen en la declaración del Combahee-River-Collective; Hill (2012) le denomina matriz de dominación; Anzaldúa (1987) habla de Bordenlans para explicitar la interconexión la raza, clase, género y sexualidad como sistemas de poder vinculados a la (in)justicia social.

La interseccionalidad como instrumento analítico, permite observar las múltiples opresiones experimentadas por las personas, y que son reproducidas por las instituciones del Estado, incluidas las leyes. Desde esta perspectiva, las normas y costumbres producen 
relaciones de poder, discriminaciones, diferencias y desigualdades entre las personas. Por ello, es clave la interseccionalidad para identificar necesidades específicas de las personas en situación de vulnerabilidad, las minorías étnicas e inmigrantes, porque son las más propensas al paro, al empobrecimiento y la exclusión, especialmente las mujeres y jóvenes migrados y racializados.

La perspectiva transnacional (Portes y Böröcz, 1998), al colocar la mirada en la persona de origen migrante consigue exhibir su heterogeneidad y sus resistencias, la forma en que conectan espacios, actúan en las estructuras de poder y desarrollan redes sociales trasnacionales (Pedone, 2010). Sin embargo, al transnacionalismo no necesariamente le interesa la dimensión cotidiana de las personas, sus restricciones o adaptaciones. Por ejemplo, no explica por qué durante la crisis económica y el deterioro del empleo, aparece rechazo, estigmatización o xenofobia contra las denominadas minorías étnicas. Este rechazo es terreno fértil para que los Estados receptores promueven leyes y legislaciones antiinmigrantes, produce segregaciones entre las personas que son nativas y las que son extranjeras (Lázaro, 2018); además genera jerarquías sociales y fronteras simbólicas que instituyen nuevas comunidades estigmatizadas y racializadas, tal como puede ocurrir con las y los hijos de personas inmigradas.

Pese a que el racismo es una realidad en Catalunya y en España, ha recibido poca atención. Consideramos que el racismo cuesta nombrarlo porque se ha construido el discurso de que el racismo es de otro tiempo, otro espacio y se lee como un acto individual. Segundo, porque la "raza" ha sido sustituida por la etnia, y los problemas raciales aparecen como sinónimo de diferencia o problema cultural, “discriminación”, estereotipo o prejuicio (Delgado, 1998).

Segato (2012), sostiene que existe una relación entre racismo, prejuicio y discriminación, ya que el prejuicio es una actitud de convicciones personales y la discriminación es el efecto de esas convicciones en la esfera pública. Ambos términos los podemos ver operando, por ejemplo, en el mundo laboral, cuando se facilita el acceso a la gente blanca para determinadas profesiones, que resultan ser las mejor remuneradas. El ejemplo demuestra que exclusión es la otra cara de un privilegio. En el presente artículo, la raza se entiende como un sistema de dominación que distribuye a la población global en posiciones, lugares y roles sociales (Lugones, 2008). Buscamos que se evite pensar a la racialización como un tema de "pigmentación", expresado en tonos de piel o de rasgos étnico-raciales. 
El rechazo a la inmigración no comunitaria en España, por parte de la población, la encontramos en el lenguaje orientado hacia la defensa de lo nacional y contra aquello/ os que ponga en riesgo al Estado de bienestar. Estos discursos generan procesos de alteridad y divide a los ciudadanos y a los no ciudadanos. Ejemplo de ello, son los derechos básicos anulados a las personas de origen inmigrante o el difícil acceso a la educación y a empleos formales, tal como se expone a continuación.

\section{Las y los jóvenes racializados y/o de origen inmigrante en España}

Las y los hijos de inmigrantes extracomunitarios, se les denomina "segundas generaciones". Si inmigrante es aquella persona que protagoniza una migración, entonces es un término que no siempre aplica a los jóvenes de las segundas generaciones, estos no necesariamente han experimentado la movilidad internacional.

Segunda generación también presenta cierto contenido racista, porque hace referencia a hijos de inmigrantes de países del "tercer mundo" o "etinificados". Para Delgado (1988, p. 115), el imaginario social atribuye lo étnico a lo premoderno, algo que es inferior. Por ello, cuando se habla de "minorías étnicas" se alude a "moros", "negros", "filipinos", "peruanos", es decir migrantes empobrecidos o de origen "gitano". Por tanto, utilizamos la expresión racializados o inmigrados para referirnos aquellos jóvenes que han nacido o han sido socializados en España.

Diversas investigaciones (García, 2003; Labrador y Blanco, 2007; Montcusí, 2007) sostienen que las y los hijos de inmigrantes heredan el estatus migratorio, se les clasifica como no autóctonos, aunque nunca hayan vivido fuera de España. Además, cargan con todos los estereotipos racistas de la migración, pues son leídos como sospecha, amenaza o portadores de "prácticas culturales inadecuadas" y se les coloca en una posición inferior. Los estados aluden a la escolarización temprana como dispositivo de asimilación (García, 2003, p. 9), e integración social. Pero, permanecer en el sistema educativo o superarlo con éxito dependerá del ambiente educativo, familiar y contextual.

Aunque se suele culpar a la familia del fracaso escolar, lo cierto es que existe una visión negativa del alumnado extranjero, se lee como no deseable (García y Olmos, 2012). Se han documentado políticas de segregación escolar encubiertas con la adscripción residencial (Cutillas y Moraes, 2018), los cuales, producen desventajas educativas para 
los estudiantes racializados (Cebolla y Garrido, 2011). Ballestín (2015), explica que al profesorado se le dificulta atender la diversidad estudiantil.

Los estereotipos y prácticas racistas las encontramos también en el mercado laboral. Suele señalarse que en la selección del personal contratado prima la calidad de los currículums, sin embargo, el proyecto "Growth, equal opportunities, migration \& markets" (University of Essex, 2018) ha demostrado que sí existe discriminación laboral. Los resultados arrojan que los candidatos de grupos étnicos minoritarios son discriminados, pese a tener la misma formación y motivación que los nacionales. La discriminación basada en el grupo afecta sobre todo en la primera fase del proceso de contratación. La investigación demuestra que las estigmatizaciones pueden desencadenar comportamiento discriminatorio y sesgar la evaluación de los candidatos al puesto de trabajo.

Lo descrito arriba, es una muestra de que en el cuerpo social el racismo, sexismo y clasismo han sido naturalizados, lo que dificulta identificarlos más allá de la discriminación. Por esta razón es necesario un análisis desde la interseccionalidad, con el fin de ver su impacto en las trayectorias educativas y laborales de las y los jóvenes racializados residentes en Barcelona. Antes, señalaremos la metodología empleada.

\section{Metodología}

Los resultados del presente trabajo derivan de la investigación denominada Horizontes titubeantes: percepciones de discriminación en las trayectorias educativas de los jóvenes hijos de inmigrantes con estudios superiores, desarrollada entre noviembre del 2019 y septiembre del 2020. Con el objetivo de explorar las percepciones de discriminación en jóvenes migrados y racializados, el estudio se basó en una metodología de corte cualitativo (Parra y Briceño, 2013). Para acercarnos a la subjetividad de las y los jóvenes, describiendo y comprendiendo su cotidianidad, se utilizó la técnica de entrevista en profundidad. Este enfoque permitió que las entrevistas se desarrollasen de una forma flexible y dinámica, creando una atmosfera calidad para que los participantes se expresaran libremente (Taylor y Bogdan, 1990).

Aunque en un inicio se planteó contar con el mayor número de jóvenes, por los efectos de la Covid-19 solamente se pudieron concretar nueve entrevistas (incluyendo a seis mujeres y tres hombres). Los criterios de selección de las y los participantes fueron que se tratase de jóvenes de entre 22 y 35 años, residentes en Barcelona, con estudios universitarios o grado superior finalizados y que fuesen hijos de padres de origen inmi- 
grante. Las y los jóvenes entrevistados son graduados o licenciados en una diversidad de disciplinas dentro de las Ciencias Sociales, el Trabajo Social, en informática, anatomía y en formaciones profesionales técnicas como la electricidad.

Las entrevistas fueron realizadas a través de la plataforma virtual Zoom y tuvieron una duración de aproximadamente 60 minutos cada una. El formato virtual de entrevista, al eliminar el uso de grabadoras, permite a las y los entrevistados expresar sus opiniones con mayor flexibilidad y libertad. Sin embargo, este formato presenta la desventaja de no permitir registrar la comunicación no verbal, la espontaneidad, los gestos, emociones o reacciones al tratar temáticas sensibles como la discriminación y el racismo.

La información producida fue analizada través del método de análisis temático. Este método reconoce las estructuraciones significativas que definen la complejidad social, que utiliza un ordenamiento esquemático y se puede realizar un análisis riguroso de los resultados (Braun y Clarke, 2006). Una vez realizado el trabajo de transcripción, los datos se organizaron de acuerdo a los siguientes temas: trayectorias familiares/migratorias, trayectorias educativas y trayectorias laborales. El análisis se centró en las experiencias de discriminación vivida por los participantes a través de la comprensión y la interpretación (Shutz, 1967). El programa informático utilizado para llevar a cabo este proceso fue Atlas.ti.

Las nueve entrevistas conforman una muestra no probabilística suficiente para capturar la percepción que las y los jóvenes mantienen sobre su realidad social, educativa y laboral. El perfil de las y los jóvenes representa la heterogeneidad descrita del sujeto estudiado manteniendo las variables de clase, género y origen. La muestra sigue los criterios de la investigación cualitativa, intensiva a pequeña escala, donde la relevancia son los casos estudiados más que la cantidad de informantes (Ragin et al., 2004).

En esta investigación el componente ético es fundamental, por ello, se entregó a cada participante el consentimiento informado, validado por miembros del Comité ético del Hospital Sant Joan de Déu. Los nombres de las y los informantes que aparecen a lo largo del artículo han sido cambiados para respetar el anonimato. 


\section{Resultados y discusión}

Fueron los procesos de regularización y reagrupación familiar, iniciados en los años 90 y 2000, los que propiciaron el aumento de menores extranjeros en colegios e institutos españoles (Mahía, 2018). La mayoría de las y los entrevistados fueron reagrupados por el padre y solo en dos casos por la madre. Los padres primero reagruparon a la madre y, años más tarde a las y los hijos. Las y los jóvenes, emigraron entre los tres y 10 años, y son de Marruecos, Republica dominicana, Ecuador y Argentina. Además, encontramos dos casos de jóvenes de descendencia marroquí nacidas en España.

El peso de la condición inmigrante, la exclusión y la estigmatización son factores que pueden limitar las aspiraciones educativas de las y los jóvenes migrados y racializados. Los jóvenes participantes, señalan que el sueño migratorio de sus padres está ligado a la obtención de mejoras laborales, económicas y educativas que beneficie a la familia. Sin embargo, los padres, a su llegada, encontrarán un ordenamiento socioeconómico que les excluye de ocupaciones, de una vivienda o del permiso de residencia o ciudadanía. Como sostiene Gil Araujo (2004) la normativa de extranjería española produce efectos clasificatorios y calificatorios entre los distintos colectivos. Según el origen o procedencia de la persona inmigrada, enfrentaran distintos requisitos para conseguir la residencia, la nacionalidad o acceder al bienestar social.

El tiempo que lleva a los padres de las y los jóvenes regularizar su estancia u obtener los trámites de reagrupación familiar, se va reformulando el proyecto migratorio y alterando la vida familiar. Por ejemplo, la mitad de las y los entrevistados, señalan no haber crecido en familias biparentales, ya sea por disolución de la pareja, por el fallecimiento de uno de los padres o por la formación de nuevas familias, tal como se aprecia a continuación.

Yo cuando llegue a los 12 años, mi madre estaba aquí con otra pareja, con el padre de mi hermano pequeño. (...) Cuando yo llegué ella ya estaba embarazada de él. (Marta, 29 años, República Dominicana)

Otro factor importante, con impacto en la educación de los hijos, es la prolongada ausencia de los padres, derivada de las largas jornadas laborales. Suele señalarse que la falta de apoyo familiar produce deserción escolar, sobre todo porque la falta de atención se debe a las duras condiciones laborales de los padres y familiares (García, 2011). Estas condiciones no están desligadas de la categoría raza. La racialización tiene un peso 
importante para que grupos de seres humanos sean insertados en una jerarquía social, laboral y política (García, 2003).

En la experiencia laboral de las personas de origen inmigrante podemos observar cómo se interrelación el género, la raza y la clase. El mercado de trabajo hace uso de las categorías para clasificar y distribuir a las personas según el trabajo, el origen y el sexo-género. En el contexto español podemos ver a mujeres de origen inmigrante empleadas en el servicio doméstico y de cuidados, un empleo que, si bien está regulado, no cuenta con cotización para la pensión, ni acceso al seguro de desempleo.

Es ilustrativo observar cómo los padres de las y los jóvenes entrevistados en España se han insertado en ocupaciones (pre)establecidas para las personas de origen inmigrante. Las madres de las y los entrevistados se han empleado en el cuidado de personas, limpieza de hogares, en fábricas o en enfermería; mientras que los padres han trabajado en la construcción, en servicios, en el comercio, en la electricidad y en el transporte. Las ocupaciones muestran una división sexual y racial del trabajo, son temporales, precarios y con largas jornadas, condiciones que, a veces, impiden a los padres tiempo para acompañar la educación formal de sus hijos/as.

Las dificultades para obtener permisos de residencia, trabajo, dominar el idioma, son factores que llevan a pensar a los inmigrantes como personas "sin estudios" o sin cualificaciones, que "acepta" todo tipo de trabajos (Labrador y Blanco, 2007, p. 79). Pero no es así. Existe un orden social donde el racismo y el patriarcado son indisolubles, y para mantenerlo es necesario el uso de mecanismos legales de diferenciación entre los nacionales y los que no lo son. La imbricación de las categorías, clase, género y origen son los que encierran a las personas en la categoría inmigrante para que cumplan distintas funciones como las laborales, las socioeconómicas o las de cohesión de la población.

En el caso de las y los jóvenes entrevistados, los padres de tres de ellos contaban con experiencia laboral en trabajos cualificados, pero en destino no se les reconoció la experiencia. Tanto para los padres cualificados como los que no, el empleo se vivirá como un "comenzar de cero", al no contar con redes de apoyo les resulta difícil la inserción laboral y les llevará a ocupaciones irregulares, mal pagados y poco cualificados.

La reproducción de desigualdades se realiza a través de un laberinto burocrático y legal para excluir y encubrir el racismo estructural con el que operan las instituciones. En el caso de España, los mecanismos legales harán inviable la homologación de títulos universitarios o de postgrado. Así lo explican las y los jóvenes entrevistados. 
Mi madre era maestra de primaria, una vez en España empezó a trabajar en el cuidado de personas mayores y en la limpieza. (Joana, 30 años, Ecuador)

El no reconocimiento de los estudios sirve como dispositivo para producir cuerpos subordinados, desechables, explotables y arrojados a la precariedad. Como lo expresa uno de los jóvenes entrevistados.

Por ejemplo, mis padres cuando llegaron a España no podían elegir y decir, bueno, no quiero trabajar en el campo o en la fábrica. (Martin, 24 años, Argentina)

En destino los padres de las y los jóvenes se enfrentan con la dificultad de compaginar trabajo, estudios y cuidados. Esta situación reduce sus posibilidades para dedicar tiempo a la supervisión de deberes escolares, lo que puede desmotivar a las y los jóvenes, y llevarlos al abandonando escolar, o a realizar alguna Formación Profesional (Cano et al., 2016). Esta es una de las razones que explican que los hijos de familias inmigradas interrumpan los estudios.

Las desigualdades sociales y de origen se ven reflejadas en el ámbito educativo, aunque el sueño de las familias sea la movilidad social a través de la educación, expresado como el "ser alguien". Lo cierto es que las y los descendientes de migrantes se encuentran en desventaja educativa, tanto por las condiciones socioeconómicas, como por las segregaciones producidas por propio sistema educativo español.

Carrasco et al. (2011), explica que en el contexto catalán la segregación concentra a infantes y adolescentes de origen extracomunitario en colegios públicos, por la dificultad que tienen las familias para afrontar los gastos y cursos extraescolares. Por tanto, las desigualdades de clase u origen no pueden ser leídas como problemas "culturales" o de inmersión lingüística.

Las y los jóvenes en sus trayectorias educativas, constantemente serán leídos como extranjeros/as y racializados. Las y los entrevistados, pese haber nacido o ser socializados como españoles/catalanes y hablar el idioma, serán leídos desde la alteridad. Las y los entrevistados continuamente tienen que escuchar el ¿tú de dónde eres?, o contestar preguntas sobre el país de origen que, a veces, suele ser una referencia lejana. Como explica Fátima.

En muchas ocasiones a las personas nativas les parece exótico o curioso tener un compañero extranjero o catalogado como extranjero. Aunque sepan que nos hemos 
educado y socializado aquí, te hacen preguntas de todo tipo sobre el país de procedencia. (Fátima, 22 años, Marruecos)

La insistencia de interrogar continuamente sobre el origen y "exotizar" como le denomina Fátima al acto de racializar, y la sorpresa expresada por parte del estudiantado nos llevan a pensar que, para muchos jóvenes locales, no es común encontrar personas inmigradas o racializadas con una trayectoria académica más allá de la básica obligatoria.

\section{Percepciones de discriminación en las trayectorias educativas}

Las y los jóvenes que han logrado llegar a la Universidad se encuentran que al título universitario habrá que sumarle otros diplomas, alargándose así la etapa educativa de los jóvenes. Las personas entrevistadas se encuentran en etapas educativas distintas, aunque todos poseen estudios superiores finalizados, la mayoría está cursando estudios de máster u otros estudios.

A pesar de que las y los jóvenes entrevistados se encuentren cursando estudios de posgrado, los resultados de esta investigación muestran trayectorias fuertemente marcadas por estigmatizaciones ligados al origen y a la condición inmigrante. Los discursos negativos, más repetidos por las y los jóvenes, son las bajas expectativas que suele tener el profesorado hacia su futuro académico, aun cuando mostraban un buen perfil.

Recuerdo las clases de orientación de $4^{\circ}$ de la ESO ${ }^{2}$ en las que decidíamos que estudiar en el siguiente curso. Entonces, la orientadora me "aconsejó" no hacer bachillerato ni optar a una carrera universitaria, aun teniendo un buen expediente académico. (Fátima, 22, Marruecos)

Sobre el alumnado de origen extranjero procedente de sectores sociales desfavorecidos y minorías culturales, constantemente se le proyecta un supuesto déficit de aprendizaje, de motivación, de trabajo. Esta percepción del docente construye al alumnado de origen extranjero extracomunitario como el "otro", como carente, sin tomar en cuenta su situación de vulnerabilidad, como apunta García (2003), se alude siempre a la supuesta teoría del déficit. Por ello, la relación que establece la escuela con el alumno de origen inmigrante es de diferenciación, de manera que al estudiantado extracomunitario poco se le incentiva para que estudien carreras universitarias.

${ }^{2}$ Educación Secundaria Obligatoria. 
Las bajas expectativas hacia el alumnado de origen extracomunitario, provoca que sea una minoría la que continúa su formación. En el caso de las y los jóvenes entrevistados, que forman parte de la minoría exitosa, argumentan que lograron el título universitario porque encontraron soporte y motivación en referentes como sus padres. Tres jóvenes mencionaron a profesores como elementos que alentaron sus trayectorias educativas. Cabe destacar que el sistema educativo español prioriza la escolarización temprana de las y los niños inmigrados, para que su integración social sea más rápida. Como se observa en el siguiente relato.

Cuando llegamos, yo no pude entrar en el colegio. Porque en ese momento yo tenía que cursar $2^{\circ}$ de la ESO. Nosotros llegamos en mayo y las clases se acaban en junio. $Y$ como yo ya iba a la ESO, en ese momento el director dijo que no hacía falta, que me podía incorporar al siguiente curso en septiembre. En cambio, mi hermano sí que fue, porque él iba a ir a primaria y dijeron que le iba a venir mejor para que se fuese integrando. (Marta, 29, República Dominicana)

La insistente alusión que se hace a los jóvenes sobre sus "orígenes o cultura”, que subraya continuamente su "otredad", su no pertenencia, produce en el alumnado la percepción de estar en los márgenes (Carrasco et al., 2011), pues, casi siempre, parten desde una posición de desventaja socioeconómica. Por tanto, la supuesta pretensión de inclusión o igualdad no puede darse en semejantes circunstancias. Las políticas educativas dirigidas a promover o impulsar la inclusión social, no abordan una parte esencial del problema material, económico y legal que repercute en las y los adolescente y sus familias, y que consiste en sentenciarles a la precarización, a la exclusión y al empobrecimiento.

Sin duda, destacamos la labor educativa como una herramienta que trasmite pensamiento crítico, habilidades para empleo y contra la adversidad. No obstante, la percepción de las y los jóvenes entorno a sus las trayectorias educativas mayoritariamente son negativas. Identifican la escuela como un medio para obtener titulaciones, pero no siempre permite acceder a un mercado laboral cada vez más competitivo y excluyente.

Y aunque no sabía lo que quería en ese momento, lo que si sabía es que no quería quedarme únicamente cursando la ESO. Bueno y laboralmente, solo con la ESO, se te cierran muchas puertas, pero sí que quería tener la opción de tenerlo ahí, aunque luego no trabajase de eso. (Andrea, 23, Ecuador) 


\section{Percepciones de discriminación y racismo en la inserción laboral}

El hecho de que las y los jóvenes cuenten con estudios superiores finalizados en el país de recepción, les posiciona con un capital social y educativo superior en comparación con sus padres. En este sentido, las y los entrevistados constatan lo que la bibliografía señala (Heath et al., 2008), que tienen cierta ventaja respecto a sus padres por el dominio del idioma, por el itinerario académico y la inmersión social.

Las y los jóvenes entrevistados, presentan distintos problemas de inserción laboral. Por ejemplo, comparten con los autóctonos la precariedad laboral, la media jornada y el desajuste entre los estudios y la ocupación. Situación que deriva de las reformas laborales del 2012, orientadas a erosionar la protección laboral, instaurando una precariedad permanente que afecta particularmente a la juventud y los grupos empobrecidos (Moreno, 2015) y produciendo mayor segmentación laboral, jerarquizado por el género, edad, origen, nivel educativo.

Al mismo tiempo tienen en común dos elementos: haber combinado los estudios superiores y/o universitarios con el trabajo asalariado, y la convicción de seguirse formando. Las y los jóvenes indican que desde los 16 años se han empleado en fruterías, restaurantes, hoteles, tiendas de ropa, en la limpieza y cuidados de personas mayores. El hecho de ser los padres su primera red de contactos, lleva a las y los jóvenes a emplearse por primera vez, en trabajos no cualificados.

Una vez obtenida la titulación universitaria o profesional, las y los jóvenes señalan que sus fuentes principales, en la búsqueda de empleo, son las páginas webs, aplicaciones de búsqueda de empleo (Infojobs o LinkedIn), redes sociales, colegios oficiales: una minoría ha recurrido a los amigos y familiares. Los resultados de la investigación señalan que el recurso del "enchufe" desaparece a la hora de buscar empleo cualificado.

Las y los jóvenes comparten con la juventud nativa la precariedad laboral, pero no compiten en igualdad de condiciones. Nuevamente la imbricación sexo-género, raza y origen la observamos en la experiencia de las y los jóvenes, se observa en la división del trabajo, en el acceso a puestos de cualificación y en la selección del personal.

Las y los jóvenes emplean el término “discriminación” para expresar el racismo cotidiano, todas/os han pasado por entrevistas de trabajo donde se les ha preguntado sobre su procedencia. Por tanto, el origen o la "cultura" es un factor que las empresas tienen en cuenta a la hora de seleccionar al personal. Aun cuando algunos jóvenes resten im- 
portancia y la consideran como una simple "curiosidad" del entrevistador; lo cierto es que el origen, la racialización y el género interrelacionados son filtros importantes a la hora de seleccionar el personal, tal como lo ha experimentado Jasmine.

Recuerdo que en la fábrica Casa Tarradellas, un sitio donde los jóvenes trabajan en verano y ganan bastante dinero, mi hermana envió el CV junto con una amiga suya. A la amiga la cogieron y a ella no. Ella estaba sorprendida, no entendía el por qué. Cuando su amiga lo pregunto en el trabajo, le dijeron que al ser una empresa que trabajan con carne de cerdo, no querían a gente musulmana. Ellos presuponían que, como no lo comemos, no podemos trabajar con carne de cerdo. (Jasmine, 25, Marruecos)

Abordar las trayectorias educativas y laborales desde la perspectiva interseccional es explorar el modo cómo las y los jóvenes inmigrados y racializados ven, internalizan, actúan y reproducen un conjunto de valores que contribuyen a perpetuar las situaciones de desigualdad. La mitad de las personas entrevistadas señalan no haberse sentido discriminadas dentro del ámbito laboral. Sin embargo, describen diferencias de trato y constantes comparaciones entre personas de un mismo origen. El trato diferenciado y el racismo en el contexto social, suele entenderse como algo individual y no como un sistema estructural.

(Discriminación) No, al menos en mi caso no, en otros compañeros sí lo he visto. $Y$ no hace mucho, hace poco en una paisana mía. Pero yo sigo pensando que todo está en la persona porque mira, las dos somos del mismo país, y mis compañeros decían Wow, qué diferencia hay entre una persona y otra, en la manera de trabajar. Todo está en la persona. (Marta, 29, República Dominicana)

En el contexto español y catalán nos encontramos con una retórica igualitarista que piensa las desigualdades como problemas "culturales", y busca remover las barreras a la interacción de las personas desmontando estereotipos y discriminaciones. Esta declaración de intenciones choca con la categorización de las personas inmigradas, a quienes se les cataloga como problemáticas y dependientes de los servicios sociales.

Por otra parte, las y los jóvenes conocen sus desventajas sociales, se saben leídos como extranjeros; sin embargo, el crecer o nacer en España les permite desenvolverse como personas locales. Situación que, a veces, les protege de agresiones racistas. Por ello, la mitad de las y los jóvenes, se resiste a reconocer las discriminaciones raciales, aunque reconocen haberse sentido inferiorizados/as en el mundo laboral. 
No creo que sea algo propio de la empresa o del equipo de recursos humanos, pienso que es más por la experiencia. Mi empresa, antes de contratarme, tuvieron malas experiencias con chicas marroquís, y sentían rechazo al coger a alguien del mismo país. Sí que después cuando empecé a trabajar, que fui la segunda opción, porque otra persona nacional rechazo el puesto, y fue cuando me llamaron a mí. Cuando empecé a trabajar, me comentaron que les había sorprendido porque se esperaban menos de mi. (Jasmín, 25, Marruecos)

La jerarquización y estigmatización impactan la subjetividad de las y los jóvenes, quienes intentaran huir de aquellos elementos negativos con los cuales se les identifica. Por ello, a las y los jóvenes entrevistados les cueste hablar del racismo; sí, lo padecen y lo detectan, pero buscan escapar de él. Pero no siempre pueden escapar a un sinnúmero de miradas, de tratos distintos, de inferiorizaciones y expresiones cotidianas que les resitúa en la alteridad.

Aunque se asocie con discriminaciones, lo que experimentan cotidianamente las y los jóvenes son expresiones del racismo, que explícitamente se atribuye a las personas en función del color, trazos físicos o grupo étnico al que la persona pertenece (Segato, 2012). Este conjunto de atributos disminuye las posibilidades de inserción laboral de las y los jóvenes.

En las entrevistas telefónicas en las que se me ha preguntado por mi origen, me ha repercutido negativamente, colgándome [el teléfono] desde el momento de conocer mi origen, o bien continuando la entrevista con poco interés.

(Fátima, 22, Marruecos)

De acuerdo con los resultados, las y los jóvenes que presenten menores oportunidades de empleo, son aquellas/os que presentan rasgos físicos asociados a las minorías, los que no tienen nacionalidad española, los que no dominan el idioma local, los que tienen religión distinta a la cristiana. Las y los entrevistados, también mencionan ser menos discriminados en comparación con sus padres. Porque manejan el lenguaje local, conocen los códigos culturales o porque son catalanes/españoles, pero no son reconocidos occidentales.

Sin la herramienta de la interseccionalidad sería difícil detectar las prácticas discriminatorias contra estos jóvenes y sus familias. Pero lo más relevante, es que nos permite ver, cómo en el cuerpo social, hay percepciones y representaciones proyectadas sobre las personas, que tienen un impacto económico, político y legal (Crenshaw, 1989). 
El racismo opera desde el Estado, porque la legislación identifica, define y clasifica a los sujetos entre ciudadanos y no ciudadanos. En esta clasificación, las y los jóvenes "heredan la condición inmigrante", por ejemplo, dos jóvenes entrevistadas mencionaron la imposibilidad de acceder a un empleo formal por no tener la tarjeta de residencia. Y los que no tienen nacionalidad española, se encuentran con la limitante de no poder ser funcionarios/as públicos.

El racismo vivido cotidianamente por las y los jóvenes en el entorno laboral, suelen ser comentarios negativos hacia las personas de origen inmigrante, y es uno de los ejemplos más repetidos por las y los entrevistados.

En mi primer trabajo que tuve, con 18 años, mis compañeros decían comentarios racistas hacia mí, o respecto a otras personas inmigrantes siempre delante de mi. (Fátima, 22 años, Marruecos)

Las jóvenes de origen marroquí dan cuenta de la racialización del género. Se encuentra ampliamente difundida la idea racista de que de que los hombres marroquíes son sospechosos, criminales y machistas, y la prueba de dicha opresión es el uso del Hiyab. La realidad es que las mujeres musulmanas suelen señalar que si no están en el mercado laboral es porque no se les contrata. Como lo demuestran la siguiente cita.

Dos de mis amigas más cercanas, una de ellas recepcionista en la ROCA ${ }^{3}$ donde entró por contactos, ella sí que lleva Hiyab, pero para trabajar no lo lleva. Y tengo otra, que estudió la carrera, tiene dos másteres. En la entrevista le dijeron que, si hubiese llevado el Hiyab, no la hubiesen cogido, pero como ella ya lo sabía no se lo puso y la cogieron. (Jasmine, 25, Marruecos)

Además del racismo en el mercado laboral, las y los jóvenes tienen que batallar con la desconfianza que asocia juventud a inexperiencia, y con los tópicos ligados a la condición inmigrante. Por ejemplo, algunos de las y los jóvenes entrevistados con plazas en el sector social, señalan haber sido elegidos para ocupar el puesto por el conocimiento de la lengua y cultura árabe en sectores donde se trabaja con población extracomunitaria. Sin embargo, esta demanda está directamente relacionada con la escasez de perfiles nacionales no poseedores de dichos conocimientos. Por tanto, son nichos de mercado en el que acceden ciertos perfiles "étnicos".

${ }^{3}$ Centro Comercial de compras outlet de lujo, ubicado a media hora de Barcelona y donde cada verano se emplea a gente joven. 
Sí, a ver en los hospitales va bien que hubiese alguien que dominase el idioma, por si habian malentendidos u otras cuestiones obvias, tenían que trabajar con moros. (Hakim, 29, Marruecos)

\section{Conclusiones}

El uso de la interseccionalidad como perspectiva de análisis nos permitió observar que si bien Catalunya, y España, presentan una población heterogénea, se tiende a pensar la diversidad como un "problema", y como un problema asociado únicamente a las poblaciones extracomunitarias. Si bien, las instituciones sociales tienen incorporado el discurso de la inclusión y la interculturalidad, sus políticas sobre la diversidad suelen fracasar, justamente porque el Estado pone en práctica una serie de dispositivos que limitan, segregan, estigmatizan y dividen a las personas entre locales y extracomunitarios.

Como se desprende de la investigación, tanto en el sistema educativo, como el mercado de trabajo, lejos de afrontar los retos de la interculturalidad, en términos de igualdad, se trata al alumnado de origen inmigrante y racializado como alteridad. A las y los jóvenes se les clasifica, identifica y ordena negativamente, basado en ideas preconcebidas que los construye de tal manera que encuentra vías para justificar las prácticas discriminatorias, tanto en los centros educativos como en el mercado laboral.

El trato diferenciador que reciben las y los jóvenes, muchas veces, fomenta una construcción identitaria negativa, por ejemplo, acerca de sus capacidades, perjudicando sus expectativas en cuanto a la continuidad y ampliación de estudios más allá de los obligatorios. En el caso de los que han logrado seguir sus estudios universitarios, tal como es el caso de las y los jóvenes del presente estudio, enfrentaran distintos retos.

En el mercado laboral, las y los jóvenes en general se enfrentan a condiciones laborales precarias e inestables, marcadas por la temporalidad y los empleos de media jornada. Esta realidad es compartida por las y los jóvenes hijos de inmigrantes, a los que también afecta los efectos negativos del mercado laboral.

Los resultados han mostrado que las trayectorias laborales de las y los jóvenes hijos de inmigrantes están condicionadas por las jerarquizaciones sociales de las personas, cuestión que inicia desde pequeños/as con el trato diferenciado que reciben en el aula, las bajas expectativas que el profesorado tiene sobre ellos/as, y que puede llevarlos al abandono escolar y a desarrollar empleos precarios y de baja cualificación. 
A pesar de que las/los jóvenes se posicionen con un capital educativo superior al de sus progenitores, el racismo estructural se manifiesta firmemente en el mercado de trabajo español. Donde se encontrarán con diversas adversidades como la imposibilidad de contratación por no poseer la nacionalidad/permiso de residencia; el descarte en los procesos de selección por compartir rasgos culturales de países del "tercer mundo"; la atribución de las competencias laborales ligados a los estigmas de origen; o la descalificación e inferiorización mediante palabras, miradas y expresiones racistas.

La interseccionalidad nos advierte que las divisiones sociales de clase, género y origen, determinadas por cada sociedad, no funcionan como entes separados, sino que se construyen y actúan juntos. En el caso de las y jóvenes entrevistados pudimos constatar cómo el origen, el género y la racialización son filtros utilizado por las empresas a la hora de contratar, ya que todos los jóvenes han referido que se les ha preguntado sobre su procedencia en el momento de la entrevista. Poseer un nombre extranjero o mostrar una fotografía con rasgos no blancos, pueden ser elementos excluyentes, a la hora de ser seleccionado para un trabajo.

Encontramos además que algunos jóvenes conocen sus desventajas en la jerarquía racial, saben que son leídos como extranjeros; pero el hecho de haber crecido o nacido en España les ha permitido protegerse, no recibir agresiones racistas físicas o verbales directas. Aunque reconocen haberse sentido inferiorizados/as en el mercado laboral, la mitad de las y los jóvenes, se resiste a reconocer el racismo estructural. Cabe destacar que a pesar de que las y los jóvenes entrevistados encuentren dificultades a la hora de acceder a un empleo, siguen invirtiendo en mejorar su curriculum profesional. Por tanto, son jóvenes que tienen altas expectativas en cuanto a su futuro, destacando en todos los casos su continua formación y búsqueda de mejores empleos.

Si bien los resultados de este estudio no son generalizables, sugieren la necesidad de implantar medidas para prevenir y combatir la discriminación empresarial frente a estos jóvenes, reduciendo sus riesgos de marginalización laboral y exclusión social y la incertidumbre sobre su situación personal y de futuro en Cataluña. 


\section{Referencias bibliográficas}

Anzaldúa, G. (1987). Borderlands/La Frontera: The New Mestiza. Spinster/Aunt Lute.

Brah, A. (2011). Cartografías de la diáspora Identidades en cuestión. Editorial Traficantes de sueños.

Braun, V. y Clarke, V. (2006). Using thematic analysis in psychology. Qualitative Research in Psychology, 3(2), 77-101. doi:10.1191/1478088706qp063oa

Ballestín, B. (2015). De "su cultura es muy fuerte" a "no se adapta a la escuela": alumnado de origen inmigrante, evaluación y efecto Pigmalión en primaria. Revista de la Asociación de Sociología de la Educación, 8(3), 361- 379. https://ojs3.uv.es/index.php/RASE/article/view/8390

Cano Hila, A. B., Sánchez, A. y Massot, M. I. (2016). Factores de riesgo y protección de las trayectorias académicas: voces y miradas del alumnado inmigrante en el contexto del área metropolitana de Barcelona, España. Revista Latinoamericana de Ciencias Sociales, Niñez y Juventud, 14(2), 1371-1387.

Carrasco, S., Pàmies J., Ponferrada, M., Ballestín, B. y Bertrám, M. (2011). Segregación escola e inmigración en Cataluña: Aproximaciones Etnográficas. En J. García Castaño y S. Carrasco Pons (Eds.), Población Inmigrante y escuela: Conocimientos y saberes de investigación (pp. 367-402). Colección Estudios Creade No 8 Ministerio de Educación.

Cebolla, H. y Garrido, L. (2011). The Impact of Immigrant Concentration in Spanish Schools: School, Class, and Composition Effects. European Sociological Review, 27(5), 606-623. https://doi.org/10.1093/esr/jcq024

Cutillas, I. y Moraes, N. (2018). Hijos de familias migrantes en espacios rurales agroindustriales de la región de Murcia: Trayectorias, pertenencias y percepciones de discriminación y rechazo. Gazeta de Antropología, 34(1).

http://www.gazeta-antropologia.es/?p=5077

Crenshaw, K. (1989). Race, Reform and Retrenchment: Transformation and Legitimation in Anti Discrimination Law. Harvard Law Review, 101, 1.331-1.387.

Davis, A. (2005). Mujer, raza y clase. Editorial Akal. 
Delgado, M. (1998). Diversitat i integració. Lògica i dinàmica de les identitats a Catalunya. Empúries.

García, I. (2003). Los hijos de inmigrantes como tema sociológico: la cuestión de la "segunda generación". Anduli: revista andaluza de ciencias sociales, 3, 27-46.

García, I. (2011). La difícil reproducción de las familias inmigrantes. ¿Hacia la formación de un proletariado étnico español? Papers: Revista de sociología, 96, 55-76.

https://papers.uab.cat/article/view/v96-n1-garcia/280

García, F. J. y Olmos, A. (2012). Segregaciones y construcción de la diferencia en la escuela. Trotta.

Gil Araujo, S. (2004). Construyendo otras. Normas, discursos y representaciones en torno a la presencia de mujeres inmigrantes no comunitarias en la Unión Europea (ponencia). VI Jornadas de Sociología, Facultad de Ciencias Sociales, Universidad de Buenos Aires, Buenos Aires.

Heath, A., Rothon, C. y Kilpi, E. (2008). The second generation in Western Europe: Education, unemployment, and occupational attainment. Annual Review of Sociology, 34, 211-235.

Hill, C. P. (2012). Rasgos distintivos del pensamiento feminista negro. En M. Jabardo (Ed.), Feminismos negros. Una antología (pp. 99-131). Editorial Traficante de Sueños.

Hill, C. P. y Bilge, S. (2019). Interseccionalidad (Roc Filella, trad.). Ediciones Morata.

Labrador, J. y Blanco, M. R. (2007). Trayectorias educativas y laborales de los jóvenes hijos de inmigrantes en España. Migraciones. Publicación Del Instituto Universitario De Estudios Sobre Migraciones, 22, 79-112. https://revistas.comillas.edu/index.php/revistamigraciones/article/view/1513

Lázaro, R. (2018). Migración circular de trabajadoras mexicanas hacia Estados Unidos: desplazamientos territoriales y subjetivos. Iberoamérica Social: revista-red de estudios sociales, IX, 55-76.

Lugones, M. (2008). Colonialidad y Género. Revista Tabula Rasa, 9, 73-101. 
Mahía, R. (2018). Población extranjera residente en España. Evolución, características, e integración económica. Anuario CIDOB de la inmigración, 11, 88-113.

https://www.cidob.org/es/articulos/anuario_cidob_de_la_inmigracion/2018/poblacion_extranjera_residente_en_espana_evolucion_caracteristicas_e_integracion_economica

Monstcusí, A. (2007). "Segundas Generaciones" ¿La inmigración como condición hereditaria? AIBR. Revista de Antropología Iberoamericana, 2(3), 459-487. ISSN: 1695-9752.

Moreno, A. (2015). La empleabilidad de los jóvenes en España: Explicando el elevado desempleo juvenil durante la recesión económica. Revista de investigación en Ciencias Sociales, 11(1), 3-20. SSN (Impresa) 2225-5117.

Ragin, C. C., Nagel, J. y White, P. (2004). The Workshop on Scientific Foundations of Qualitative Research. National Science Foundation. http://bit.ly/3XEND3

Parra, M. y Briceño, I. (2013). Aspectos éticos de la investigación cualitativa. Enfermería Neurológica, 12(3), 118-121.

Pedone, C. (2010). Cadenas y redes migratorias: propuesta metodológica para el análisis diacrónico-temporal de los procesos migratorios. EMPIRIA, Revista de Metodología de Ciencias Sociales, 19, 101-132. ISSN: 1139-5737.

Portes, A., Aparicio, R. y Haller, W. (2018). Hacerse adulto en España: La integración de los hijos de inmigrantes. En E. Mañé (Ed.), Inmigración y asilo, en el centro de la arena política (pp. 149-181). CIDOB.

Portes, A. y Böröcz, J. (1998). Migración contemporánea. Perspectivas teóricas sobre sus determinantes y sus modalidades de incorporación. En Graciela Malgessini (Ed.), Cruzando fronteras. Migraciones en el sistema mundial (pp. 43-73). Icaria-Fundación Hogar del empleado..

Segato, R. (2012). Racismo, discriminación y acciones afirmativas: Herramientas conceptuales. Observatorio Jurídico Constitucional, 5, 1-16.

https://portal.idp.emnuvens.com.br/observatorio/article/view/686

Schutz, A. (1967). The phenomenology of the social world (G. Walsh y F. Lehnert, trad.). North Western University Press (original publicado en 1932). 
Taylor, S. J. y Bogdan, R. (1990). Introducción a los métodos cualitativos de investigación. Paidós.

http://mastor.cl/blog/wpcontent/uploads/2011/12/Introduccion-a-metodos-cualitativos-de-investigación-Taylor-y-Bogdan.-344-pags-pdf.pdf

Universidad Carlos II de Madrid. (2019). Un estudio analiza la discriminación laboral a nivel europeo.

https://www.uc3m.es/ss/Satellite/UC3MInstitucional/es/Detalle/Comunicacion _C/1371270248618/1371216001705/Un_estudio_analiza_la_discriminacion_laboral_a_nivel_europeo

University of Essex and The European Union's Horizon 2020. (2018). Grow, Equal Opportunities, Migration and Markets: GEMM PROJECT in Focus 2015-2018.

https://gemm2020.eu/wp-content/uploads/2018/11/GEMM-Project_In_Focus.pdf

\section{Biografía de las autoras}

\section{Josselyn Urdiales Correa}

Graduada en Trabajo Social en la Universidad de Barcelona, Máster en Estudios Avanzados en Exclusión Social por la Universidad de Barcelona, España. Correo electrónico: josselynurdiales@coltscat.cat

ORCID ID: 0000-0002-0242-5294

\section{Rosa Lázaro Castellanos}

Doctora en Antropología Social por la Universidad de Barcelona, además cuenta con una Maestría en Estudios del Desarrollo por el Colegio de Postgraduados y tiene una licenciatura en Economía por la Benemérita Universidad Autónoma de Puebla. Actualmente es Profesora Asociada en el Departamento de Antropología Social de la Universidad de Barcelona. Correo electrónico: rosylazaro@ub.edu ORCID ID: 0000-0003-2969-3958 